\title{
X-Ray Interferometric Solution of the Surface Registration Problem
}

\author{
G. Materlik \\ Hamburger Synchrotronstrahlungslabor, Deutsches Elektronen-Synchrotron, D-2000 Hamburg 52, Germany \\ and \\ A. Frahm \\ II. Institut für Experimentalphysik, Universität Hamburg, D-2000 Hamburg 50, Germany \\ and \\ M. J. Bedzyk \\ Hamburger Synchrotronstrahlungslabor, Deutsches Elektronen-Synchrotron, D-2000 Hamburg 52, Germany
} (Received 22 November 1983)

\begin{abstract}
A Bonse-Hart $\mathrm{x}$-ray interferometer was used to determine the (220) Fourier component of the distribution function of a chemisorbed submonolayer of bromine on a (111) silicon surface. This measurement demonstrates not only the presence of an x-ray interference field above the crystal surface of the analyzer of a Laue-case interferometer, but when coupled with Bragg-case standing-wave measurements also provides the means to determine uniquely the position of surface atoms relative to the bulk lattice.
\end{abstract}

PACS numbers: $68.20 .+\mathrm{t}, 61.10 . \mathrm{Fr}$

$\mathrm{X}$-ray dynamical diffraction has been used recently to determine the position of adsorbed surface atoms on a perfect-crystal substrate. ${ }^{1}$ The potentials of this method are best illustrated by reminding the reader that under certain geometrical conditions two photon Bloch-wave eigenstates are allowed solutions in a dynamically diffracting crystal. Each has two partial waves with wave vectors $\vec{K}_{0}$ and $\vec{K}_{H}=\vec{K}_{0}+\vec{H}$, where $\vec{H}$ is the reciprocal-lattice vector of the reflecting set of Miller planes. Therefore, the spatial periodicity of the standing-wave field equals that of the diffraction planes, and one photon Bloch wave has nodes of the electric field distribution which coincide with the maxima of the $(h, k, l)$ Fourier component of the charge-density distribution. The second solution has antinodes at the equivalent places and its nodes halfway between.

Changing the incidence condition by tuning the reflection angle of a crystal through the Bragg condition in a Bragg-case geometry, when incident and reflected waves are lying on the same side of the entrance surface, causes a continuous transition of the excitation of one Bloch-wave solution to the other. This means a movement of the standing-wave field in the $-\overrightarrow{\mathrm{H}}$ direction by one-half of a diffraction-plane spacing from being in phase with the $(h, k, l)$ charge-density component to being out of phase by $\pi$ rad. By measuring the fluorescence yield due to the decay of the photon-excited $1 s$ hole, a process which is proportional to the electric field intensity at the sites of the nuclei, Batterman ${ }^{2,3}$ was able to verify this theory. Later Golovchenko, Batterman, and $\mathrm{Brown}^{4}$ used the same technique to determine positions of foreign atoms in the direction of $\overrightarrow{\mathrm{H}}$. Although this technique is restricted to Braggcase measurements, one can obtain projections of displacements of atoms in the surface plane by reflecting from diffraction planes which are not parallel to the surface and provide the basis for a triangulation technique. ${ }^{5}$ For general cases this requires a careful consideration of the geometrical window function on the acceptance side of the crystal.

We shall show in this paper that a Laue-case geometry, when entrance and exit surfaces of the incident and diffracted beams are different, allows a direct determination of atomic positions in a plane parallel to the surface. As is well known, standing interference patterns can be created by summing the amplitudes of two coherent plane waves. While this interference can be realized easily with optical waves, $x$ rays have such short wavelength and small coherence length that one has to take recourse to crystal diffraction in order to generate two coherent wave trains. Therefore, Bonse and Hart, ${ }^{6}$ ingeniously used the Laue case of diffraction to excite two spatially separated coherent beams and were thereby able to manipulate their phases independently. This principle was used to construct successfully the first $x$-ray interferometer. ${ }^{6,7}$ The initial crystal splits the incident beam into two coherent waves. A second crystal intercepts each of these beams and acts as Laue-case mirror to change the beam direction and to lead both beams to interference again. A third crystal finally 
serves to analyze the spatial distribution of the standing electric field component of the resulting interference pattern.

Unlike the Bragg case of diffraction, the nodal and antinodal planes of this standing-wave pattern lie perpendicular to the surface and a phase difference, induced between the incident beams will cause a shift of the pattern parallel to the surface plane. Also in a Laue-case reflection, two Blochwave eigenstates of the photon inside the crystal are excited simultaneously at each angular position of a scan through the reflection condition. This causes interference which gives rise to "pendellösungs effects. ${ }^{8}$ " However, close to the surface of the analyzer of a Laue-case interferometer, the interference of two incident waves produces a standing-wave field with antinodal planes lying halfway between the scattering planes if the phase difference is zero, and a change of the relative phase by $\pi$ rad will move these planes in a continuous manner by half a diffraction-plane constant.

The strategy of a position determination in the plane of the surface is similar to a Bragg-case standing-wave measurement. An adsorbate is deposited on the surface of the analyzer of an $x-$ ray interferometer. The phase difference between the two incident waves is changed and the fluorescence from the bulk and surface atoms are registered simultaneously by a $\mathrm{Si}(\mathrm{Li})$ solidstate detector.

The experiment was carried out by using the instrument ROEMO at the Hamburger Synchrotron Radiation Laboratory (HASYLAB) at DESY in Hamburg. The storage ring DORIS running at 3.7 $\mathrm{GeV}$ electron energy with a medium current of about $60 \mathrm{~mA}$ served as the source of synchrotron $\mathrm{x}$ radiation (SXR).

The inset in Fig. 1 shows the experimental arrangement. The white SXR spectrum was initially monochromatized with a slightly dispersive double-crystal monochromator which consisted of a symmetric germanium (220) and an asymmetric silicon (220) crystal. Because of the different refractive indices of silicon versus germanium, the higher-order contamination is reduced considerably. ${ }^{10}$ The first crystal also enhances the total intensity throughput because of the higher germanium electron density. This increased emittance is utilized effectively by the second asymmetric silicon crystal, the acceptance of which at $15.4 \mathrm{keV}$ is increased by a factor of 3.5 to serve as a plane-wave generator for the outgoing beam with a resulting width of 0.5

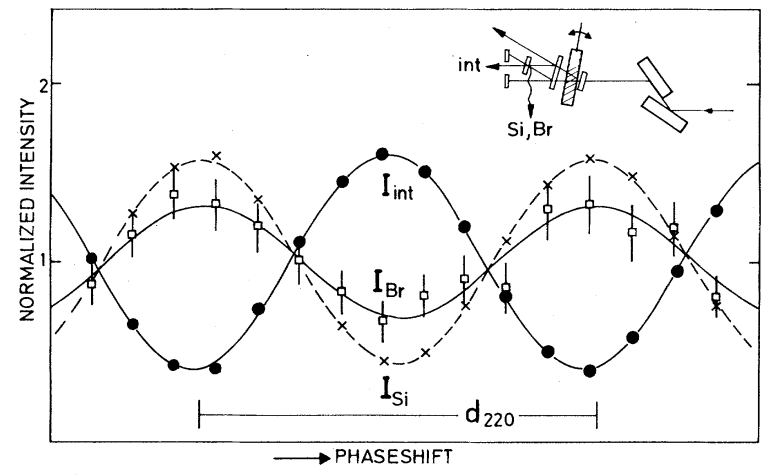

FIG. 1. Phase scan of the fluorescence yields from near-surface silicon atoms $\left(I_{S_{i}}\right)$ and from adsorbed $\mathrm{Br}$ atoms $\left(I_{\mathrm{Br}}\right)$ relative to the transmitted intensity $\boldsymbol{I}_{\text {int }}$. The inset shows schematically the experimental setup in a side view.

arc sec. This width is small in comparison to the (220) Laue-case reflection width of 2.6 arc sec.

The plane-wave-like beam is divided in two coherent beams by the beam splitter of a monolithic Laue-case Bonse-Hart $\mathrm{x}$-ray interferometer. Both beams then pass through a parallelsided Lucite phase shifter which can be rotated by a stepper motor in order to change the relative phases of these beams. This difference is zero when the plate is oriented parallel to the mirror surface and the rotation then produces a $\pi$ rad phase shift by a rotation of $2^{\circ} .{ }^{11}$ As shown in Fig. 1, four beams exist from behind the second mirror and two of them are brought to interference again in the region in front of the analyzer crystal. The intensity in one of the noninterfering rays served, after registration by a $\mathrm{NaI}$ detector, as a reference signal to stabilize the angular alignment of the interferometer relative to the monochromator. An analog feedback servo system $^{12}$ was used for this control as well as for keeping the monochromator crystals in a stable alignment. A second $\mathrm{NaI}$ detector registered the intensity of the forward-scattered interference beam (int). The $\mathrm{Si}(\mathrm{Li})$ solid-state detector looked under grazing incidence at the front surface of the analyzer to collect the fluorescence scattering from the bulk $(\mathrm{Si})$ and the adsorbate $(\mathrm{Br})$ atoms.

The interferometer was cut from one dislocation-free silicon block with three mirrors standing up, each with a thickness of $1.5 \mathrm{~mm}$. The surface was oriented parallel to (111) Miller planes with (220) diffraction planes oriented perpendicular to the surface. After chemical etch- 
ing of the whole interferometer the analyzer surface was cleaned by a brief Syton polish. The crystal was then dipped again in $\mathrm{HF}$ to remove oxide layers and finally bromine was adsorbed from a wet methanol solution. During a measurement the interferometer was enclosed in a housing for thermal stabilization and the built-in moiré effect was partly compensated by electric heating from the side of the $\mathrm{Si}(\mathrm{Li})$-detector cool tip.

The result of a phase scan is shown in Fig. 1 for the intensity of the interfering beam behind the interferometer and for the fluorescence yields from the substrate and from the adsorbate. The total coverage of $\mathrm{Br}$ atoms in this run was about $\frac{1}{4}$ of a monolayer as was determined by comparison to an implanted standard sample.

The intensity transmitted through the analyzer is out of phase by about $\pi$ rad relative to the fluorescence yield from the silicon atoms close to the front surface. This demonstrates the Borrmann effect ${ }^{13}$ since only the wave field with antinodal planes of the electrical field intensity halfway between the diffraction planes reaches the back plane of the analyzer, while fluorescence from atoms near the entrance surface is maximized when the antinodes overlap with the inner core-level states.

The $\mathrm{Br}$ fluorescence yield relative to the photon flux which was incident on the first interferometer mirror was about $3 \times 10^{-8}$. Even for the collimated high photon intensity from DORIS this only resulted in a count rate of $0.3 \mathrm{Br} K_{\alpha}$ photons per second and per square millimeter of the illuminated analyzer surface. The total counting time needed for the set of data shown in Fig. 1 was 160

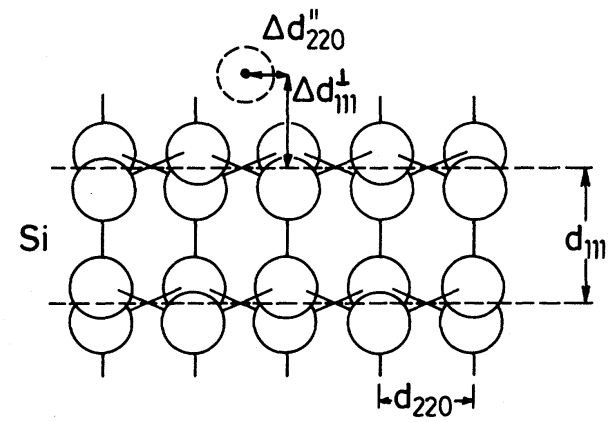

FIG. 2. Arrangement of silicon atoms close to the (111) surface. Dashed lines give the (111) and (220) diffraction planes. $\Delta d_{111}{ }^{\perp}$ and $\Delta d_{220}$ " are the distance of the adsorbed atom (dashed circle) determined by a Bragg-case and by an interferometric measurement, respectively. min with a sensitive detector area of $30 \mathrm{~mm}^{2}$ at about $5 \mathrm{~mm}$ distance from the radiating spot.

The fluorescence yield from the $\mathrm{Br}$ adsorbate follows the substrate emission with a relative phase shift $\varphi_{220}{ }^{\prime \prime}=(-0.008 \pm 0.018) 2 \pi \mathrm{rad}$. The contrast of this curve indicates the fraction of $\mathrm{Br}$ atoms which register coherently with the lattice parallel to the surface plane and this was determined to be $f_{c, 220} "=0.57 \pm 0.04 .{ }^{14}$

These measured values represent the phase and the amplitude of the first Fourier coefficient of the bromine distribution function relative to the (220) Fourier component of the substrate charge density. If the $\mathrm{Br}$ atoms are displaced relative to the (220) planes (see Fig. 2) in a single-sited position this result means that $57 \%$ of all adsorbed atoms register coherently with the (220) planes in a position $\Delta d_{220} "=0.014 \pm 0.034 \AA$. This value indicates a substitutional position on the (220) netplanes; however, Fig. 3 shows that three different, highly symmetric positions $(A, B$, and $C)$ are still possible adsorption sites. All three positions lie on intersections of (220) planes and can, therefore, not be distinguished by one single distance measurement in the [220] direction.

To triangulate this position we have also performed a Bragg-case measurement relative to the (111) diffraction planes to determine the distance $\Delta d_{111}$ as illustrated in Fig. 2. For this measurement we have again used SXR from DORIS and the instrument is described elsewhere. ${ }^{15}$ Using the same chemisorption procedure as described above resulted in a phase $\varphi_{111}{ }^{\perp}=(0.82$ $\pm 0.02) 2 \pi \mathrm{rad}$ and an amplitude $f_{c, 111}{ }^{\perp}=0.77 \pm 0.01$

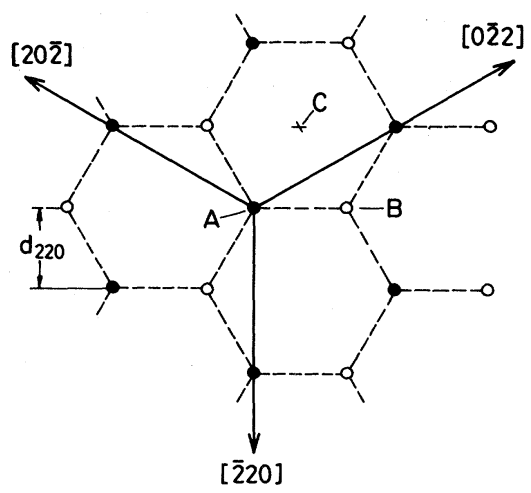

FIG. 3. Arrangement of Si atoms in the (111) surface of the analyzer relative to the three sets of (220) planes with the spacing $d_{220}$. Closed and open circles indicate first-layer and second-layer atoms, respectively. $A$, $B$, and $C$ are symmetric positions lying on intersections of $(220)$ planes. 
for the first Fourier coefficient of the $\mathrm{Br}$ distribution function in the (111) direction. Under the assumption of one single adsorption site this means that $77 \%$ of the $\mathrm{Br}$ atoms occupy positions with a displacement $\Delta d_{111}{ }^{\perp}=2.57 \pm 0.06 \AA$ which is in close agreement with earlier measurements. ${ }^{1,5}$

Summing the atomic radii in a covalent bonding of the $\mathrm{Si}$ and $\mathrm{Br}$ ato $\mathrm{ms}$ gives a $\mathrm{Si}-\mathrm{Br}$ bond length of $2.28 \AA$. On the other hand, $\Delta d_{111}{ }^{\perp}$ is measured relative to the (111) diffraction planes as shown in Fig. 2 and this implies for position $A$ a $\mathrm{Si}-\mathrm{Br}$ distance of $2.18 \pm 0.06 \AA$ which is in good agreement with the covalent bond length. ${ }^{16}$ The values for the distances which are inferred from position $\boldsymbol{B}$ and $\boldsymbol{C}$, however, are in distinctive disagreement with the value which is obtained from the sum of the atomic radii.

The comparison of the coherent fractions also shows reasonable agreement. Whether the remaining difference of about $15 \%$ is caused by a distribution function of the $\mathrm{Br}$ in the (111) plane, relative to the (220) diffraction planes, with more than one adsorption site, or whether the bonding angle in position $A$ is not the same as in the bulk, which makes from symmetry arguments several places in the (111) plane equivalent and will reduce the coherent fraction accordingly, cannot be answered from our present results. Measurements of higher order $\mathrm{s}^{15}$ and determinations of different Fourier components such as (422) in a (111) plane, however, can uniquely ${ }^{17}$ answer the se questions.

One of us (G.M.) gratefully acknowledges many helpful discussions with J. A. Golovchenko in the early stages of this project.

${ }^{1}$ P. L. Cowan, J. A. Golovchenko, and M. F. Robbins, Phys. Rev. Lett. $\underline{44}, 1680$ (1980).

${ }^{2}$ B. W. Batterman, Phys. Rev. 133, A759 (1964).

${ }^{3}$ B. W. Batterman, Phys。 Rev. Lett. 22, 703 (1969).

${ }^{4} \mathrm{~J}$. A. Golovchenko, B. W. Batterman, and W. L. Brown, Phys. Rev. B 10, 4239 (1974).

${ }^{5}$ J. A. Golovchenko, J. R. Patel, D. R. Kaplan, P. L. Cowan, and M. J. Bedzyk, Phys. Rev. Lett. $\underline{49}$, 560 (1982).

${ }^{6} \mathrm{U}$. Bonse and M. Hart, Appl. Phys. Lett. $\underline{6}, 155$ (1965).

${ }^{7} \mathrm{U}$. Bonse and M. Hart, Z. Phys. 188, 154 (1965).

${ }^{8}$ This means, for example, that the standing-wavefield modulation becomes zero at certain depths within the crystal.

${ }^{9}$ J. A. Golovchenko, D. R. Kaplan, G. Materlik, and P. Funke, to be published.

${ }^{10}$ U. Bonse, G. Materlik, and W. Schröder, J. Appl. Crystallogr. 9 , 223 (1976).

${ }^{11}$ Note that this requires a much less precise phase drive than for a Bragg-case measurement, where the corresponding phase shift occurs over the width of a reflection curve.

${ }^{12}$ A. Krolzig, G. Materlik, M. Swars, and J. Zegenhagen, to be published.

${ }^{13} \mathrm{G}$. Borrmann, Z. Phys. $\underline{42}, 157$ (1941).

${ }^{14}$ This value was already corrected for a loss of contrast which is caused by the built-in moiré effect.

${ }^{15} \mathrm{G}$. Materlik and J. Zegenhagen, to be published.

${ }^{16}$ Note that the positional accuracy of such measurement can still be increased if needed (Ref. 15).

${ }^{17}$ Obviously, a unique position determination needs more than two distance measurements. 